\title{
Melatonin administration reduces inflammatory pain in rats
}

This article was published in the following Dove Press journal:

Journal of Pain Research

17 September 2012

Number of times this article has been viewed

\author{
Gabriela Laste ${ }^{1-3}$ \\ Isabel Cristina de Macedo 1,3 \\ Joanna Ripoll Rozisky ${ }^{1-3}$ \\ Fernanda Ribeiro da Silva ${ }^{1,3}$ \\ Wolnei Caumo ${ }^{1,2}$ \\ Iraci LS Torres ${ }^{1-3}$ \\ 'Laboratório de Farmacologia da Dor, \\ Departamento de Farmacologia, \\ Instituto de Ciências Básicas da \\ Saúde, ${ }^{2}$ Programa de Pós-Graduação \\ em Medicina, Ciências Médicas, \\ Universidade Federal do Rio Grande \\ do Sul, Porto Alegre, Brazil; ' ${ }^{3}$ nidade \\ de Experimentação Animal e Grupo \\ de Pesquisa e Pós-Graduação, \\ Hospital de Clínicas de Porto Alegre, \\ Porto Alegre, Brazil
}

Correspondence: Iraci Lucena da Silva Torres

Departamento de Farmacologia, ICBS, UFRGS, Rua Sarmento Leite, 500 sala 202, 90050-170, Porto Alegre, RS, Brazil Tel +55 5l 33083183

Fax +55 5l 3308 3।2I

Email iracitorres@gmail.com

\begin{abstract}
In view of the broad range of effects attributed to melatonin, this study evaluated its analgesic effect on inflammatory pain induced by complete Freund's adjuvant (CFA) in Wistar rats. Inflammation was induced by intradermal CFA injection in the hind paw of all animals, which were then divided into two groups that received either $60 \mathrm{mg} / \mathrm{kg}$ of melatonin or vehicle ( $1 \%$ alcohol in saline), intraperitoneally, for three days. The analgesic effect of melatonin was assessed by the hot-plate test, immediately and thereafter at 30,60, 90, and 120 minutes after the first administration and 24 hours after once-daily administration for 2 more days. After CFA injection, melatonin administration increased withdrawal latency at 60 minutes after the first dose. After the end of treatment, melatonin showed a significant analgesic effect on inflammatory pain. This study paves the way for exploration of how brief courses of treatment could improve this analgesic effect in the late phases of inflammatory pain.
\end{abstract}

Keywords: analgesic response, complete Freund's adjuvant, hot-plate test, inflammation, melatonin, nociception

\section{Introduction}

Melatonin (N-acetyl-5-methoxytryptamine) is a hormone synthesized and secreted by the pineal gland. This indolamine is derived from the amino acid L-tryptophan and plays an important role in the regulation of physiological functions, including sleep and circadian rhythms. ${ }^{1,2}$ Previous studies have suggested that melatonin has a variety of pharmacological effects. ${ }^{3}$ Among the broad range of effects attributed to melatonin, its potential antinociceptive and anti-inflammatory actions have been studied in an animal model of acute pain. ${ }^{4}$ The analgesic properties of melatonin have been demonstrated in previous studies conducted by our group ${ }^{5,6}$ and in other studies of patients with fibromyalgia. ${ }^{7,8}$ Melatonin administration has been found to raise pain thresholds. ${ }^{9}$ In humans, melatonin helps regulate circadian rhythms and, in addition to its analgesic action, also exerts hypnotic, ${ }^{10}$ anxiolytic, ${ }^{10}$ anti-inflammatory, ${ }^{11}$ and antioxidant effects. ${ }^{9}$ To date, the precise mechanisms through which melatonin attenuates nociception have not been elucidated. Its analgesic effect has been shown to involve MT1 and MT2 receptors present in the spinal cord and various brain regions. ${ }^{9,12}$ Another study suggested that the opioid system may be involved in this effect. ${ }^{3}$ In inflammatory pain, the mechanism of melatonin analgesia appears to be by inhibition of nitric oxide production, reducing activation of the transcription factor NF- $\mathrm{KB}$ (nuclear factor kappa $\mathrm{B}$ ), the expression of cyclooxygenase and prostaglandins, and the recruitment of polymorphonuclear cells to the site of inflammation. ${ }^{4,11}$ However, little is known about the antinociceptive effects of melatonin from its acute, short-term administration. In this context, the present study 
sought to characterize the antinociceptive effect of melatonin when given in a single acute dose and over a 3-day course of treatment in Wistar rats subjected to inflammatory pain induced by complete Freund's adjuvant (CFA).

\section{Methods and materials}

Male Wistar rats, 60 days old and weighing 250-300 g, were used. The animals were housed, four to five per cage, in $49 \times 34 \times 16 \mathrm{~cm}$ polypropylene home cages with sawdustcovered floors. Animals were kept on a standard 12-hour dark/light cycle (lights on between 0700 and 1900 hours), at room temperature $\left(22^{\circ} \mathrm{C} \pm 2{ }^{\circ} \mathrm{C}\right)$, with access to food and water ad libitum. Animal handling and all experiments were performed in accordance with international guidelines for animal welfare. The protocol for this experimental study was approved by the Research Ethics Committee of the institution where the work was conducted (GPPG-HCPA 100013, approved March 8, 2010), and it adhered to the ethical and methodological standards of the Guide for the Care and Use of Laboratory Animals (NIH Publication No 85-23, revised 1985), the UK Animals (Scientific Procedures) Act of 1986, and the European Communities Council Directives of November 24, 1986 (86/609/EEC).

CFA and melatonin were purchased from Sigma-Aldrich (St Louis, MO). Melatonin was dissolved in 1\% ethanol in saline (v/v) immediately before use and administered at a dose of $1 \mathrm{~mL} / \mathrm{kg}$.

The rats were acclimated to the maintenance room for 1 week before the start of the experiment. All animals received intradermal injections of CFA into the right hind paw to induce inflammation. Animals were randomly allocated to one of two groups ( $n=11-12$ per group): melatonin (treatment) or vehicle (control). Rats in the experimental group were treated with intraperitoneal injections of melatonin $(60 \mathrm{mg} / \mathrm{kg}$; final solution $60 \mathrm{mg} / \mathrm{mL}),{ }^{13,14}$ while those in the control group received vehicle ( $1 \%$ ethanol in saline). Injections were administered 1 hour post-CFA and once daily for 2 more days thereafter (for a total of 3 days of melatonin administration), always at 1500 hours. Hot-plate measurements were performed at baseline (before CFA injection), 1 hour after CFA injection, and 30, 60, 90, and 120 minutes and 72 hours after the first administration of melatonin or vehicle, to assess the cumulative effects.

CFA-induced inflammation is commonly recommended as an acute and chronic animal inflammation model for the study of therapeutic approaches to pain. ${ }^{8}$ Briefly, animals were anesthetized with isoflurane, and inflammation was induced by a single intradermal injection of $100 \mu \mathrm{l} \mathrm{CFA}$ (heat-killed Mycobacterium tuberculosis suspended in paraffin oil, plus mannide monooleate, $1 \mathrm{mg} / 1 \mathrm{~mL}$ ) into the right hind paw.

The hot-plate test was carried out to assess the effects of melatonin on the thermal nociceptive threshold. Twenty-four hours prior to testing, all rats were given 5 minutes to acclimate to the hot plate. The temperature of the plate was kept at $55^{\circ} \mathrm{C}$. The animals were placed in glass funnels onto the heated surface. With a stopwatch, the experimenter recorded, for the latency of response in seconds, the time between placement of the animals and the onset of paw licking or jumping behavior. ${ }^{15}$

\section{Results}

The results are presented in Figure 1 as mean \pm standard deviation. The time course of the latency response on the hotplate test was plotted using a repeated measures analysis of variance (ANOVA), followed by a Bonferroni post hoc test, with time as the repeated measure and the group as the independent variable. Student's $t$-test for independent samples was used to ascertain whether the mean hot-plate latencies at baseline and after CFA injection were similar for both groups. $P$-values of $<0.05$ were considered significant.

There was no between-group difference in latency at baseline (V: $4.7 \pm 0.6$ seconds; M: $4.5 \pm 0.5$ seconds; Student's $t$-test, $P=0.10$ ) or after CFA injection (V: $2.5 \pm 0.6$ seconds; M: $2.7 \pm 1.3$ seconds; Student's $t$-test, $P=0.18$ ). The repeated measures ANOVA (Figure 1) results showed a time effect $\left(\mathrm{F}_{(1,23)}=22.8, P=0.001\right)$, a group effect $\left(\mathrm{F}_{(1,23)}=25.0\right.$, $P=0.001)$, and an interaction between time and group $\left(\mathrm{F}_{(1,23)}=12.5, P=0.001\right)$. The post hoc Bonferroni test showed an effect from melatonin both after 60 minutes and after 3 days of treatment ( $P<0.05$ for both), but no effect at 30 minutes, 90 minutes, or 120 minutes $(P>0.05$ for all) (30 minutes: $2.7 \pm 0.1$ seconds; 60 minutes: $3.7 \pm 0.3$ seconds;

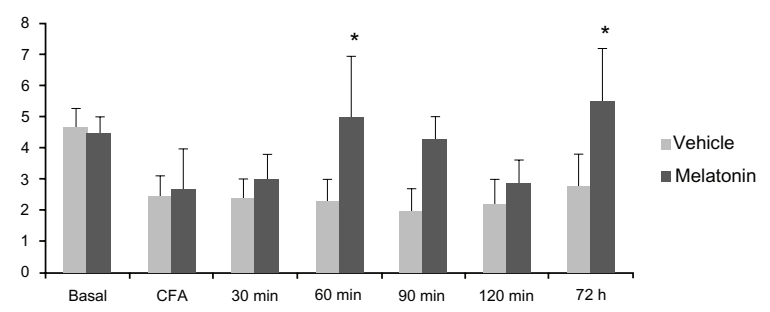

Figure I Analgesic effect of melatonin on inflammatory pain based on hot-plate latency.

Notes: Data presented as the mean \pm standard deviation (SD) as latency of response in seconds to onset of paw licking or jumping. *Significant between-group difference (repeated measures analysis of variance [ANOVA] with post hoc Bonferroni test; $\mathrm{P}<0.05, \mathrm{n}=1 \mathrm{I}-12$ /group).

Abbreviations: CFA, complete Freund's adjuvant; min, minutes; h, hours. 
90 minutes: $3.1 \pm 0.1$ seconds; 120 minutes: $2.6 \pm 0.1$ seconds; $4.1 \pm 0.3$ seconds).

\section{Discussion}

The results of the present study suggest that melatonin plays a role in increasing the threshold of pain associated with CFAinduced inflammation. Our findings are consistent with those of a previous study showing that, at higher doses $(60 \mathrm{mg} / \mathrm{kg}$ and $120 \mathrm{mg} / \mathrm{kg}$ ), melatonin produced effective antinociception in the tail-flick test, starting 15 minutes after melatonin administration and peaking after 30 minutes, with the effect lasting over 100 minutes. ${ }^{16}$ In addition, a previous study of intrathecal melatonin administration showed antiallodynic activity in rats with neuropathic pain, with the effect observed from 30 minutes through 240 minutes after administration. ${ }^{17}$ In diabetic rats, the best antinociceptive effect was observed with 60 minutes' pretreatment $(300 \mathrm{mg} / \mathrm{kg}$ orally) in the formalin test. ${ }^{3}$

The inflammatory pain model employed in this study has been widely used to test several analgesic drugs, ${ }^{18}$ including drugs with anti-inflammatory activity. ${ }^{8}$ Tissue damage or inflammation leads to the release of a variety of inflammatory mediators - such as histamine, serotonin, arachidonic acid metabolites (via cyclooxygenase), and kinin - from leukocytes migrating to the affected region. ${ }^{19}$ This cascade can be induced by CFA injection. CFA-induced inflammation has been shown to increase levels of cytokines and free radicals. ${ }^{20}$ Some of these agents activate nociceptors, while others release local algogenic agents. Algogenic substances sensitize nociceptive neurons, thereby enhancing neuronal excitability in pain transmission pathways and causing secondary hyperalgesia. ${ }^{21,22}$ The transmission of pain by primary afferent fibers, the central processes of which are located in the dorsal horn of the spinal cord, is thought to be influenced by several neurotransmitter systems, including opioid, adrenergic, substance P, glutamate, and neurokinin receptors. ${ }^{21,22}$

It should be noted that we used the hot-plate test with the specific intent of evaluating centrally acting analgesics, as described by Woolfe and MacDonald. ${ }^{15}$ This test is a neurogenic-modulated model that produces, at a constant temperature, two types of behavioral response (paw licking and jumping), both of which are considered to be supraspinally integrated. ${ }^{15}$

This result suggests a probable central effect of melatonin, as has been described by other authors. ${ }^{2,9}$ The possible mechanisms of action for the antinociceptive effect of melatonin include activation of supraspinal sites ${ }^{16}$ and inhibition of "spinal windup." 23 Thus, the reduction in secondary inflammatory hyperalgesia in our study could indicate that melatonin reduces the excitability of pain transmission in dorsal horn neurons by acting on membrane-bound MT1 and MT2 receptors. ${ }^{9}$ There is also experimental evidence to suggest that the analgesic effect of melatonin is mediated by opioids ${ }^{1}$ and by gamma-aminobutyric acid (GABAergic) systems. ${ }^{17}$

While it has been suggested that melatonin has antiinflammatory properties, we cannot rule out that its central antinociceptive effect is mediated by these mechanisms alone. It should be noted that we did not measure paw edema or biochemical markers of inflammation, and thus cannot infer any improvement in inflammation. Furthermore, melatonin has been shown to produce a marked anti-inflammatory effect at peripheral sites by inhibiting the release of proinflammatory cytokines and thereby relieving pain. ${ }^{24,25}$ Several studies in rat models of inflammation have demonstrated that another possible mechanism of melatonin effect in inflammatory diseases is inhibition of nitric oxide production, modulation of activation of the transcription factor NF-kB, reduced expression of cyclooxygenase and prostaglandins, and recruitment of polymorphonuclear cells at the site of inflammation. ${ }^{4,18}$

\section{Conclusion}

This study showed that melatonin treatment exerts an antihyperalgesic effect in an animal model of inflammatory pain. This finding paves the way for exploration of how short courses of treatment could improve analgesia in the late phases of inflammatory pain.

\section{Acknowledgments}

This study was supported by the following Brazilian funding agencies: National Council for Scientific and Technological Development, CNPq (Dr ILS Torres, Dr W Caumo); HCPA/ PIBIC/CNPq (FR da Silva): Committee for the Development of Higher Education Personnel, CAPES (JR Rozisky, G Laste); Graduate Research Group of Hospital de Clínicas de Porto Alegre (Dr ILS Torres; Grant 100013).

\section{Disclosure}

The authors report no conflicts of interest in this work. The authors alone are responsible for the content and writing of the paper.

\section{References}

1. Ambriz-Tututi M, Granados-Soto V. Oral and spinal melatonin reduces tactile allodynia in rats via activation of MT2 and opioid receptors. Pain. 2007;132(3):273-280.

2. Shin DJ, Jeong CW, Lee SH, Yoon MH. Receptors involved in the antinociception of intrathecal melatonin in formalin test rats. Neurosci Lett. 2011;494:207-210. 
3. Arreola-Espino R, Urquiza-Marín H, Ambriz-Tututi M, et al. Melatonin reduces formalin-induced nociception and tactile allodynia in diabetic rats. Eur J Pharmacol. 2007;577:203-210.

4. Esposito E, Paterniti I, Mazzon E, Bramanti P, Cuzzocrea S. Melatonin reduces hyperalgesia associated with inflammation. J Pineal Res. 2010;49:321-331

5. Caumo W, Torres F, Moreira NLJR, et al. The clinical impact of preoperative melatonin on postoperative outcomes in patients undergoing abdominal hysterectomy. Anesth Analg. 2007;105:1263-1271.

6. Caumo W, Levandovski R, Hidalgo MP. Preoperative anxiolytic effect of melatonin and clonidine on postoperative pain and morphine consumption in patients undergoing abdominal hysterectomy: a double-blind, randomized, placebo-controlled study. J Pain. 2009;10:100-108.

7. Wilhelmsen M, Amirian I, Reiter RJ, Rosenberg J, Gögenur I. Analgesic effects of melatonin: a review of current evidence from experimental and clinical studies. J Pineal Res. 2011;51:270-277.

8. Zaringhalam J, Akbari A, Tekieh E, Manaheji H, Rezazadeh S. Achllea santolina reduces serum interleukin-6 level and hyperalgesia during complete Freund's adjuvant-induced inflammation in male Wistar rats. Chin J Integr Med. 2010;12:1180-1189.

9. Srinivasan V, Pandi-Perumal SR, Spence DW, et al. Potential use of melatonergic drugs in analgesia: mechanisms of action. Brain Res Bull. 2010;81:362-371.

10. Garzón C, Guerrero JM, Aramburu O, Guzmán T. Effect of melatonin administration on sleep, behavioral disorders and hypnotic drug discontinuation in the elderly: a randomized, double-blind, placebo-controlled study. Aging Clin Exp Res. 2009;21:38-42.

11. Bilici D, Akpinar E, Kiziltunç A. Protective effect of melatonin in carrageenan-induced acute local inflammation. Pharmacol Res. 2002;46:133-139.

12. Zahn PK, Lansmann T, Berger E, Speckmann EJ, Musschoff U. Gene expression and functional characterization of melatonin receptors in the spinal cord of the rat: implications for pain modulation. J Pineal Res. 2003;35:24-31.

13. Mickle A, Sood M, Zhang Z, Shahmohammadi G, Sengupta JN. Antinociceptive effects of melatonin in a rat model of post-inflammatory visceral hyperalgesia: a centrally mediated process. Pain. 2010;149: 555-564.
14. Ray M, Mediratta PK, Mahajan P, Sharm KK. Evaluation of the role of melatonin in formalin-induced pain response in mice. Indian J Med Sci. 2004;58:122-130.

15. Woolfe G, MacDonald AD. The evaluation of the analgesic action of pethidine hydrochloride. J Pharmacol Exp Ther. 1944;80:300-307.

16. Yu CX, Zhu CB, Xu SF, Cao XD, Wu GC. The analgesic effects of peripheral and central administration of melatonin in rats. Eur $J$ Pharmacol. 2000;403:49-53.

17. Wu FS, Yang YC, Tsai JJ. Noncompetitive inhibition of the glycine receptor-mediated current by melatonin in cultured neurons. Brain Res. 2000;881:208-211.

18. Lichtenberger LM, Romero JJ, Dial EJ, Moore JE. Naproxen-PC: a GI safe and highly effective anti-inflammatory. Inflammopharmacology. 2008;5:17-21

19. Maleki N, Garjani A, Nazemlyeh H, et al. Potent anti-inflammatory activities of hydroalcoholic extract from aerial parts of Stachys inflata on rats. Ethnopharmacol. 2001;75:213-218.

20. Zaringhalam J, Manaheji H, Mghsoodi N, Farokhi B, Mirzaiee V. Spinal mu-opioid receptor expression and hyperalgesia with dexamethasone in chronic adjuvant arthritis rats. Clin Exp Pharmacol Psysio. 2008;35:1309-1315.

21. Poeggeler B, Reiter RJ, Hardeland R, Tan DX, Barlow-Walden LR. Melatonin and structurally related endogenous indoles act as potent electron donors and radical scavengers in vitro. Redox Rep. 1996;2: 179-184.

22. Tan DX, Chen LD, Poeggeler B, Manchester LC, Reiter RJ. Melatonin: a potent, endogenous hydroxyl radical scavenger. Endocr J. 1993;1: 57-60.

23. Morgan PJ, Barret P, Howell E, Helliwell R. Melatonin receptors: localization, molecular pharmacology and physiological significance. Neurochem Int. 1994;24:101-146.

24. Lewy AJ, Emens J, Jackman A, Yuhas K. Circadian uses of melatonin in humans. Chronobiol Int. 2006;23:403-412.

25. Yoon MH, Park HC, Kim WM, et al. Evaluation for the interaction between intrathecal melatonin and clonidine or neostigmine on formalin induced nocicetion. Life Sci. 2008;83:845-850.
Journal of Pain Research

\section{Publish your work in this journal}

The Journal of Pain Research is an international, peer-reviewed, open access, online journal that welcomes laboratory and clinical findings in the fields of pain research and the prevention and management of pain. Original research, reviews, symposium reports, hypothesis formation and commentaries are all considered for publication.

\section{Dovepress}

The manuscript management system is completely online and includes a very quick and fair peer-review system, which is all easy to use. Visit http://www.dovepress.com/testimonials.php to read real quotes from published authors. 